\title{
Social, economic and demographic correlates of overweight and obesity in primary-school children: preliminary data from the Healthy Growth Study
}

\author{
George Moschonis, Sofia Tanagra, Anastasia Vandorou, Aikaterini E Kyriakou, \\ Vasiliki Dede, Paraskevi E Siatitsa, Alexandra Koumpitski, Odysseas Androutsos, \\ Evangelia Grammatikaki, Maria Kantilafti, Anthi Naoumi, Aliki E Farmaki, \\ Aikaterini Siopi, Eleni Z Papadopoulou, Elpida Voutsadaki, Fanouria Chlouveraki, \\ Konstantina Maragkopoulou, Efstathoula Argyri, Aggeliki Giannopoulou and Yannis Manios* \\ Department of Nutrition \& Dietetics, Harokopio University of Athens, 70 El. Venizelou Avenue, \\ 17671 Kallithea, Athens, Greece
}

Submitted February 2009: Accepted May 2010

\begin{abstract}
Objective: To record the prevalence of overweight and obesity in urban primaryschool children in relation to several socio-economic and demographic factors. Design: Cross-sectional.

Setting/subjects: A representative sample of 729 schoolchildren (379 male and 350 female), aged 9-13 years, stratified by parental educational level, was examined in the urban region of Athens. Weight and height were measured using standard procedures. The International Obesity Task Force thresholds were used for the definition of overweight and obesity. Several socio-economic and demographic data and the child's 'popularity' score were also recorded with specifically designed standardized questionnaires.

Results: The prevalence of overweight and obesity was $29 \cdot 6 \%$ and $11 \cdot 1 \%$, respectively. Annual family income of $€ 12000-20000(\mathrm{OR}=1 \cdot 58)$, residence ownership (OR $=1.63)$ and the grandmother as the child's primary caregiver $(\mathrm{OR}=1 \cdot 38)$ were significantly associated with higher odds of childhood overweight and obesity. Non-Greek parental nationality $(\mathrm{OR}=0.72)$ and higher 'popularity' scores of children $(\mathrm{OR}=0 \cdot 42)$ were significantly associated with lower odds of overweight and obesity. The grandmother as the child's primary caregiver and an annual family income of $€ 12000-20000$ remained significantly associated with childhood overweight and obesity after adding all significant correlates of childhood overweight and obesity observed at the bivariate level in a multivariate regression model ( $\mathrm{OR}=1 \cdot 51$ and $1 \cdot 61$, respectively).

Conclusions: Among family income, residence ownership, child's primary caregiver, parental nationality and popularity scores that were identified as significant correlates of childhood overweight and obesity at the bivariate level, lower family income and grandmother as the child's primary caregiver were the only factors that remained significantly associated with childhood overweight and obesity at a multivariate level.
\end{abstract}

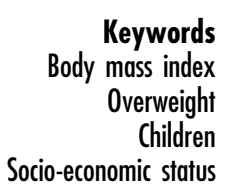

The prevalence of overweight and obesity among Greek children has increased over the past decades ${ }^{(1)}$. The rates of childhood overweight and obesity in Greece are similar or slightly higher to those reported in other European countries $^{(2,3)}$. As with adults ${ }^{(4)}$, the adoption of adverse obesogenic behaviours by children seem to have a socio-economic, demographic and cultural dimension ${ }^{(5,6)}$. Parental educational level, family income, ethnicity and the child's primary caregiver are some of the socio-economic and demographic factors that have been previously suggested to modify children's energy balance behaviours (i.e. dietary and physical activity patterns), thus having either a negative or positive impact on childhood obesity $^{(6,7)}$. More specifically, previous studies in Greece and other developed countries have reported that paternal and/or maternal educational level was inversely associated with childhood obesity ${ }^{(8-10)}$. Furthermore, several indices that reflect the family's socio-economic status, such as annual family income, house or car ownership, house size, etc. have also been reported to be inversely 
related to childhood obesity ${ }^{(11)}$. Nationality has also been reported to exert a significant effect on childhood obesity, although the limited data available in the literature for European countries provide some contradictory findings on their exact association ${ }^{(10,11)}$.

Clearly, family and other social factors influence children's eating patterns that may subsequently influence the onset of obesity. As children comprise the adult population of tomorrow, recording and understanding the prevalence of obesity in young children and the social, economic and demographic factors related to the phenomenon can facilitate the development of effective public health intervention policies in counteracting obesity and its health consequences later in life. It is known that early prevention is more effective in managing the epidemic of obesity in comparison with treating obesity later in life ${ }^{(12)}$.

The aim of the present study was to record the prevalence of overweight and obesity among 9-13-year-old schoolchildren in the capital city of Athens, the largest urban area of Greece, in relation to several socio-economic and demographic characteristics of children and their families. To test our research hypothesis, the preliminary data from an urban sub-cohort of the 'Healthy Growth Study' were used in the present analyses.

\section{Subjects and methods}

\section{Sampling}

The 'Healthy Growth Study' is a cross-sectional study initiated in May 2007. Approval to conduct the study was granted by the Greek Ministry of National Education and the Ethics Committee of Harokopio University of Athens. The population under study comprised schoolchildren aged 9-13 years attending the fifth and sixth grades from primary schools located in municipalities within the wider region of Athens. The sampling of municipalities and schools in the present study was random, multi-stage and stratified by the parental educational level and the total population of pre-adolescent students, thus yielding a representative sample of primary-school children from the wider urban region of Athens. More specifically, the municipalities in the county of Attica were divided into three groups based on the average educational level of their adult population (25-65-year-olds) that was estimated from data provided by the National Statistical Service of Greece (Census 2001). This procedure yielded two parental education cut-off points that allowed us to categorize municipalities into three categories of different socio-economic levels (SEL), i.e. higher, medium and lower SEL. Consequently, based on data from the National Statistical Service of Greece, a certain number of municipalities, proportional to the size of their pre-adolescent population (9-14-year-olds), was randomly selected from each of these three SEL groups. Finally, an appropriate number of schools was randomly selected from each of these municipalities in relation to the population of schoolchildren registered in the fifth and sixth grades in each municipality, based on data obtained from the Greek Ministry of Education.

Weight and height were measured in all pupils attending the fifth and sixth grades in the participating primary schools as part of a school-based health and nutrition education programme. Full medical examination (i.e. anthropometrics and body composition measurements, blood collection, clinical examination, etc.) and questionnaire data were obtained from a sub-group of pupils whose parents signed a relative consent form. More specifically, an extended letter explaining the aims of the present study and a consent form for conducting full measurements were provided to all parents or guardians having a child in these schools. Parents who responded positively had to sign the consent form and provide their contact details. Signed parental consent forms for full measurements were collected for 754 out of 1236 children (response rate: $61 \%$ ). From the total number of positive responses, complete medical examination data became available for 729 children who will be reported as 'study subjects' throughout the paper. Regarding data on weight and height, these were obtained from 1201 children in total (i.e. 729 'study subjects' and 472 'weight and height participants').

\section{Antbropometry}

'Study subjects' and 'weight and height participants' from each primary school underwent an examination by two trained members of the research team. The protocol and equipment used for all measurements were the same for all regions. The physical examination included basic anthropometric measurements, i.e. weight and height. Body weight was recorded to the nearest $10 \mathrm{~g}$ using a Seca digital scale (Seca Alpha, Model 770, Hamburg, Germany) and with children standing without shoes in the minimum clothing possible. Height was measured to the nearest $0 \cdot 1 \mathrm{~cm}$ using a commercial stadiometer (Leicester Height Measure, Invicta Plastics Ltd, Oadby, UK) with the participants not wearing shoes, their shoulders in a relaxed position, their arms hanging freely and their head aligned in the Frankfort plane ${ }^{(13)}$. Weight and height were converted to BMI using Quetelet's equation (weight $(\mathrm{kg}) /$ height $^{2}\left(\mathrm{~m}^{2}\right)$ ). The International Obesity Task Force (IOTF) cut-off points ${ }^{(14)}$ were used to categorize participants as 'underweight', 'normal weight', 'overweight' and 'obese'.

\section{Socio-economic and demographic variables}

Data on the socio-economic and demographic background of the families having at least one child participating in the study were collected from the parents (most preferably from the mother) during scheduled face-to-face interviews at the school site. For those parents not able to attend the meetings (approximately $5 \%$ of the total sample), data were collected via telephone interviews. All interviews were conducted by a research team of ten members who were rigorously trained to minimize interviewer's effect with 
the use of a standardized questionnaire. More specifically, the data collected by parents included (i) demographic characteristics, such as parent's age, educational level (years of education), origin and nationality; (ii) economic characteristics, such as family mean annual income over the past 3 years ( $€$ /year), family residence ownership; (iii) social characteristics, such as family size, i.e. the number of family members living in the family residence; the primary caregiver(s) of the child, i.e. parents, grandparents, nanny or someone else; family type based on parental marital status, i.e. core families and single-parent families with parents who were not married, widowed or divorced. Furthermore, a 'popularity' score among classmates was developed for each child by asking all pupils in the same class, including 'weight and height participants', to name at least one of their three best friends in their class. All votes were then summed to create 'popularity' scores for all 'study subjects'. These scores were then adjusted for class size by dividing them by the total number of boys and girls attending the same class for male and female 'study subjects', respectively. The test-retest reliability of the 'popularity score' index was checked in a representative sub-sample of 100 pupils and was found to be quite high, reaching a rate of $94 \cdot 8 \%$.

\section{Statistical analysis}

Some of the independent variables used in the present analyses were continuous while others were categorical. Normality of the distribution of continuous variables was analysed using the Kolmogorov-Smirnov test. Normally distributed continuous variables were expressed as mean and SD, skewed variables were reported as median (25th and 75th interquartiles) and categorical variables were reported as frequencies (\%). Associations between continuous and categorical variables were examined using Student's $t$ test for normally distributed variables and the non-parametric Mann-Whitney $U$ test for skewed variables, while the association among the categorical variables was assessed using the $\chi^{2}$ test. In order to test the effect of the independent variables examined on being overweight and obese, univariate logistic regression analyses were conducted and crude OR with 95\% CI were computed. Furthermore, adjusted OR and 95\% CI were also estimated using multivariate logistic regression analysis, including all variables that were found to be significantly $(P<0 \cdot 05)$ associated with childhood overweight and obesity at a univariate level. Moreover, since the sample of the present study is based on a cluster design (all children in primary schools enrolled in the study), this was taken into account in multiple logistic regression analysis using the option 'robust cluster'. All reported $P$ values were based on two-sided tests. The level of statistical significance was set at $P<0 \cdot 05$. The Statistical Package for Social Sciences statistical software pakage version $13 \cdot 0$ (SPSS Inc., Chicago, IL, USA) was used for all statistical analyses and the STATA statistical software package version 8 (STATA Corp., College Station, TX, USA) for testing the clustering effect of schools.

\section{Results}

Table 1 displays the prevalence of underweight, normal weight, overweight and obesity for all pupils (i.e. both 'study subjects' and 'weight and height participants') attending the fifth and sixth grades in the schools under study. Overall, the observed prevalence was 3.5\% for underweight, $57 \cdot 7 \%$ for normal weight, $27 \cdot 9 \%$ for overweight and $10.9 \%$ for obesity. No significant differences were observed between 'study subjects' and 'weight and height participants' with respect to these prevalence rates $(P=0 \cdot 270)$, thus reducing the chance of any potential bias deriving from non-participation in the full protocol of the present study. Table 1 also summarizes the differences in the prevalence of underweight, normal weight, overweight and obesity among male and female 'study subjects'. Specifically, the prevalence of obesity was found to be significantly higher in male than female subjects $(15 \cdot 1 \%$ v. $6.6 \%, P<0.001)$. Although a higher prevalence of underweight $(3 \cdot 1 \%$ v. $1 \cdot 4 \%)$ and normal weight $(60 \cdot 3 \%$ v. $53.9 \%$ ) was observed in female than male subjects, this was not significantly differentiated between genders.

The socio-economic and demographic characteristics of the population of 'study subjects' are presented in Table 2. In summary, the mean age of children under study was $11 \cdot 2(\mathrm{SD} 0 \cdot 7)$ years. Approximately half of the children's population under study was living in medium SEL residential areas while their parents had a medium educational level (9-14 years). Nationality in the majority of subjects was

Table 1 Prevalence of overweight and obesity in study subjects and weight and height participants

\begin{tabular}{|c|c|c|c|c|c|c|c|c|c|c|}
\hline \multirow[b]{3}{*}{ Weight groups } & \multicolumn{6}{|c|}{ Study subjects ( $n$ 729) } & & & & \\
\hline & \multicolumn{2}{|c|}{$\begin{array}{l}\text { Male } \\
(n \text { 379) }\end{array}$} & \multicolumn{2}{|c|}{$\begin{array}{l}\text { Female } \\
(n \text { 350) }\end{array}$} & \multicolumn{2}{|c|}{$\begin{array}{l}\text { Total } \\
(n 729)\end{array}$} & \multicolumn{2}{|c|}{$\begin{array}{l}\text { Weight and height participants } \\
\qquad(n \text { 472) }\end{array}$} & \multicolumn{2}{|c|}{$\begin{array}{l}\text { All subjects } \\
(n \text { 1201) }\end{array}$} \\
\hline & $n$ & $\%$ & $n$ & $\%$ & $n$ & $\%$ & $n$ & $\%$ & $n$ & $\%$ \\
\hline Underweight & 7 & $1 \cdot 8$ & 16 & $4 \cdot 6$ & 23 & 3.2 & 19 & $4 \cdot 0$ & 42 & 3.5 \\
\hline Normal weight & 201 & $53 \cdot 0$ & 208 & $59 \cdot 4$ & 409 & $56 \cdot 1$ & 284 & $60 \cdot 2$ & 693 & $57 \cdot 7$ \\
\hline Overweight & 113 & $29 \cdot 8$ & 103 & $29 \cdot 4$ & 216 & $29 \cdot 6$ & 119 & $25 \cdot 2$ & 335 & $27 \cdot 9$ \\
\hline Obese & 58 & $15 \cdot 1$ & 23 & $6 \cdot 6^{*}$ & 81 & $11 \cdot 1$ & 50 & $10 \cdot 6$ & 131 & $10 \cdot 9$ \\
\hline
\end{tabular}

${ }^{*} P<0.001$ for differences between genders derived from the $\chi^{2}$ test. 
Table 2 Socio-economic status and demographic characteristics of study subjects

\begin{tabular}{|c|c|c|}
\hline \multirow[b]{2}{*}{ Age (years)* } & \multicolumn{2}{|c|}{$\begin{array}{l}\text { Study subjects } \\
\quad(n \text { 729) }\end{array}$} \\
\hline & $11 \cdot 2$ & $0 \cdot 7$ \\
\hline Popularity' scoret & 0.33 & $0 \cdot 16-0.60$ \\
\hline \multirow[t]{2}{*}{ Family size (number of family members) } & $4 \cdot 0$ & $3 \cdot 0-4 \cdot 0$ \\
\hline & $n$ & $\%$ \\
\hline \multicolumn{3}{|l|}{ SES of residential area } \\
\hline Lower & 254 & $34 \cdot 7$ \\
\hline Medium & 314 & $43 \cdot 0$ \\
\hline Higher & 161 & $22 \cdot 3$ \\
\hline \multicolumn{3}{|l|}{ Annual family income ( $€ /$ year) } \\
\hline$<12000$ & 200 & $27 \cdot 6$ \\
\hline $12000-20000$ & 211 & $28 \cdot 9$ \\
\hline $20000-30000$ & 159 & $21 \cdot 8$ \\
\hline$>30000$ & 159 & $21 \cdot 8$ \\
\hline \multicolumn{3}{|l|}{ Fathers' age categories } \\
\hline Lower tertile $(<41$ years old $)$ & 251 & $34 \cdot 5$ \\
\hline Medium tertile ( $41-46$ years old) & 270 & $37 \cdot 0$ \\
\hline Higher tertile ( $>46$ years old) & 208 & $28 \cdot 5$ \\
\hline \multicolumn{3}{|l|}{ Mothers' age categories } \\
\hline Lower tertile ( $<37$ years old) & 300 & $41 \cdot 2$ \\
\hline Medium tertile ( $37-42$ years old) & 239 & $32 \cdot 8$ \\
\hline Higher tertile ( $>42$ years old) & 190 & $26 \cdot 0$ \\
\hline \multicolumn{3}{|l|}{ Paternal educational level } \\
\hline Low ( $\leq 9$ years of education) & 158 & $21 \cdot 7$ \\
\hline Medium ( $>9$ and $\leq 14$ years of education) & 353 & $48 \cdot 4$ \\
\hline High ( $>14$ years of education) & 218 & $29 \cdot 9$ \\
\hline \multicolumn{3}{|l|}{ Maternal educational level } \\
\hline Low ( $\leq 9$ years of education) & 128 & $17 \cdot 5$ \\
\hline Medium ( $>9$ and $\leq 14$ years of education) & 382 & $52 \cdot 4$ \\
\hline High ( $>14$ years of education) & 219 & $30 \cdot 0$ \\
\hline \multicolumn{3}{|l|}{ Parents' nationality } \\
\hline Greeks & 516 & $70 \cdot 9$ \\
\hline Non-Greeks & 213 & $29 \cdot 1$ \\
\hline \multicolumn{3}{|l|}{ Residence ownership } \\
\hline No & 268 & $36 \cdot 6$ \\
\hline Yes & 461 & $63 \cdot 4$ \\
\hline \multicolumn{3}{|l|}{ Child's primary caregiver } \\
\hline Parents & 448 & $61 \cdot 5$ \\
\hline Grandfather & 47 & $6 \cdot 4$ \\
\hline Grandmother & 192 & $26 \cdot 3$ \\
\hline Nanny/someone else & 42 & $5 \cdot 8$ \\
\hline \multicolumn{3}{|l|}{ Parental marital status } \\
\hline Core families - married parents & 641 & $87 \cdot 9$ \\
\hline Single parent families - not married parents & 16 & $2 \cdot 2$ \\
\hline Single parent families - widowed parent & 14 & $1 \cdot 9$ \\
\hline Single parent families - divorced parents & 58 & $7 \cdot 9$ \\
\hline
\end{tabular}

SES, socio-economic status.

*Data are presented as mean and SD.

tData are presented as median and 25 th-75th percentiles.

Greek (70.9\%). Furthermore, most children (63.4\%) lived in residences owned by their families. The grandmother was the child's primary caregiver in almost $25 \%$ of our population. An analysis per gender revealed that parent's nonGreek nationality and residence ownership were higher for girls than boys $(32.5 \%$ v. $26 \%, P<0 \cdot 05 ; 67 \cdot 2 \%$ v. $59 \cdot 7 \%$, $P<0 \cdot 05$, respectively), whereas the grandmother as the child's primary caregiver was more frequent for girls rather than boys $(28.8 \% v \cdot 23.5 \%, P<0.05)$, and the nanny or someone else appeared more frequently as the child's primary caregiver for boys than girls $(8 \cdot 3 \% v .3 \cdot 5 \%, P<0 \cdot 05)$.

Table 3 summarizes the crude OR and $95 \%$ CI for being overweight and obese also controlling for the clustering effect of schools. An annual family income of $€ 12000$ 20000 was related to an increased likelihood of childhood overweight and obesity (OR $=1 \cdot 56 ; 95 \%$ CI $1 \cdot 07,2 \cdot 35)$ compared to an annual family income of $>€ 30000$. Furthermore, children whose residence was owned by their family were $1 \cdot 68$ times more likely ( $95 \%$ CI $1 \cdot 22,2 \cdot 25$ ) to be overweight and obese than children whose residence was not owned by their family. Moreover, in families in which the primary caregiver of the child was his/her grandmother, the risk of childhood overweight and obesity was 1.38 times higher $(95 \%$ CI $1.03,1.98)$ than families where the child's primary caregivers were his/her parents. In contrast, children whose parents' nationality was Greek were 1.38 times less likely (95\% CI 1.03, 1.96) to be overweight and obese than children whose parents' nationality was non-Greek. Finally, for every $1^{\circ}$ increase in the child's 'popularity' score, the likelihood of childhood overweight and obesity was found to decrease by $60 \%$ (95\% CI $0 \cdot 17,0 \cdot 97)$. Furthermore, Table 3 also presents the adjusted OR and 95\% CI derived from the multivariate logistic regression analysis, including in the same model all variables that were found to be significantly related to childhood overweight and obesity $(P<0.05)$ at a univariate level. Based on the results derived from the present analysis, those variables that remained significantly associated with childhood overweight and obesity were the grandmother as the child's primary caregiver (OR $=1 \cdot 53 ; 95 \% \mathrm{CI} 1 \cdot 05$, $2 \cdot 29)$ and annual family income of $€ 12000-20000$ (OR $=1 \cdot 61 ; 95 \%$ CI $1 \cdot 03,2 \cdot 61)$, respectively.

\section{Discussion}

The findings of the present study revealed a high prevalence of both overweight and obesity in a population of primary-school children living in a large urban area of Greece. Using the international cut-off points recommended by the IOTF ${ }^{(14)}$ (Table 2), 29.6\% of children were found to be overweight and $11 \cdot 1 \%$ were found to be obese with the prevalence of obesity being significantly higher for boys than girls $(15 \cdot 1 \% v \cdot 6 \cdot 6 \%)$. Comparing the findings of the present study regarding the prevalence of overweight and obesity with those reported for other European countries $^{(3)}$, there seems to be a geographic variation. Precisely, the prevalence reported from studies conducted with children living in southern European countries surrounding the Mediterranean, including the present study, ranged between $20 \%$ and $41 \%$ and was considerably higher than the prevalence reported for the northern areas in Europe that showed prevalence rates in the range of $10-20 \%{ }^{(3)}$. Several potential explanations have been proposed for these North-South trends in the prevalence of childhood overweight in Europe including higher stature and resting energy expenditure for children living in northern latitudes and a genetic predisposition towards weight gain in children living in southern Europe ${ }^{(3)}$. 
Table 3 Crude and adjusted OR and $95 \% \mathrm{Cl}$ for the association between overweight and obesity with several socio-economic and demographic factors in the study subjects $(n$ 729)

\begin{tabular}{|c|c|c|c|c|c|}
\hline & \multicolumn{5}{|c|}{ Overweight and obese } \\
\hline & \multirow[b]{2}{*}{$\%$} & \multicolumn{2}{|c|}{ Crude } & \multicolumn{2}{|c|}{ Adjusted } \\
\hline & & OR & $95 \% \mathrm{Cl}$ & $\mathrm{OR}^{\star}$ & $95 \% \mathrm{Cl}$ \\
\hline \multicolumn{6}{|l|}{ Annual family income (€/year) } \\
\hline$>30000$ & $38 \cdot 5$ & $1 \cdot 00$ & Ref. & $1 \cdot 00$ & Ref. \\
\hline$<12000$ & 33.9 & $0 \cdot 81$ & $0 \cdot 54,1 \cdot 27$ & $1 \cdot 05$ & $0 \cdot 61,1 \cdot 86$ \\
\hline $12000-20000$ & $49 \cdot 7$ & 1.56 & $1 \cdot 07,2.35$ & $1 \cdot 61$ & $1 \cdot 03,2 \cdot 61$ \\
\hline $20000-30000$ & $41 \cdot 0$ & 1.06 & $0 \cdot 70,1 \cdot 79$ & $1 \cdot 02$ & $0.62,1.67$ \\
\hline \multicolumn{6}{|l|}{ Parents' nationality } \\
\hline Non-Greek & $35 \cdot 8$ & $1 \cdot 00$ & Ref. & $1 \cdot 00$ & Ref. \\
\hline Greek & $44 \cdot 1$ & $1 \cdot 38$ & $1.03,1.96$ & $1 \cdot 04$ & $0.65,1.68$ \\
\hline \multicolumn{6}{|l|}{ Residence ownership } \\
\hline No & $34 \cdot 4$ & $1 \cdot 00$ & Ref. & $1 \cdot 00$ & Ref. \\
\hline Yes & $46 \cdot 7$ & 1.68 & $1 \cdot 22,2 \cdot 25$ & $1 \cdot 58$ & $0 \cdot 98,2 \cdot 20$ \\
\hline \multicolumn{6}{|l|}{ Child's primary caregiver } \\
\hline Parents & $39 \cdot 0$ & $1 \cdot 00$ & Ref. & $1 \cdot 00$ & Ref. \\
\hline Grandfather & $48 \cdot 8$ & $1 \cdot 43$ & $0 \cdot 74,2 \cdot 85$ & $1 \cdot 66$ & $0.83,3.19$ \\
\hline Grandmother & $46 \cdot 9$ & 1.38 & $1.03,1.98$ & 1.53 & $1 \cdot 05,2 \cdot 29$ \\
\hline Nanny/someone else & $45 \cdot 9$ & $1 \cdot 34$ & $0 \cdot 67,2 \cdot 63$ & $1 \cdot 62$ & $0 \cdot 82,3 \cdot 10$ \\
\hline $\begin{array}{l}\text { 'Popularity' score (adjusted for class participation by gender) } \\
\text { SES of residence region }\end{array}$ & & $0.65,1.84$ \\
\hline Lower & $38 \cdot 6$ & $1 \cdot 00$ & Ref. & - & - \\
\hline Medium & $42 \cdot 8$ & $1 \cdot 15$ & $0 \cdot 81,1 \cdot 62$ & & \\
\hline Higher & $39 \cdot 9$ & $1 \cdot 05$ & $0 \cdot 72,1 \cdot 58$ & & \\
\hline \multicolumn{4}{|l|}{ Fathers' age categories } & - & - \\
\hline Lower tertile $(<41$ years old $)$ & $43 \cdot 6$ & $1 \cdot 00$ & Ref. & & \\
\hline Medium tertile (41-46 years old) & $37 \cdot 9$ & $0 \cdot 80$ & $0 \cdot 57,1 \cdot 14$ & & \\
\hline Higher tertile ( $>46$ years old) & $44 \cdot 0$ & $1 \cdot 00$ & $0 \cdot 66,1 \cdot 48$ & & \\
\hline \multicolumn{4}{|l|}{ Mothers' age categories } & - & - \\
\hline Lower tertile $(<38$ years old $)$ & $44 \cdot 4$ & $1 \cdot 00$ & Ref. & & \\
\hline Medium tertile (38-42 years old) & $40 \cdot 1$ & $0 \cdot 83$ & $0 \cdot 57,1 \cdot 18$ & & \\
\hline Higher tertile ( $>42$ years old) & $40 \cdot 1$ & $0 \cdot 81$ & $0 \cdot 55,1 \cdot 22$ & & \\
\hline \multicolumn{4}{|l|}{ Paternal educational level } & - & - \\
\hline Low ( $\leq 9$ years of education) & $39 \cdot 1$ & $1 \cdot 00$ & Ref. & & \\
\hline Medium ( $>9$ and $\leq 14$ years of education) & $45 \cdot 5$ & $1 \cdot 26$ & $0 \cdot 78,1 \cdot 92$ & & \\
\hline High ( $>14$ years of education) & $36 \cdot 6$ & 0.93 & $0.59,1.46$ & & \\
\hline \multicolumn{4}{|l|}{ Maternal educational level } & - & - \\
\hline Low ( $\leq 9$ years of education) & $38 \cdot 6$ & $1 \cdot 00$ & Ref. & & \\
\hline Medium ( $>9$ and $\leq 14$ years of education) & $42 \cdot 7$ & $1 \cdot 17$ & $0 \cdot 74,1 \cdot 80$ & & \\
\hline \multirow{2}{*}{\multicolumn{4}{|c|}{ Parental marital status }} & & \\
\hline & & & & - & - \\
\hline Core families - married parents & $41 \cdot 6$ & $1 \cdot 00$ & Ref. & & \\
\hline Single parent families - not married parents & $53 \cdot 3$ & $1 \cdot 50$ & $0 \cdot 52,4 \cdot 26$ & & \\
\hline Single parent families - widowed parent & $53 \cdot 8$ & $1 \cdot 61$ & $0.52,4.91$ & & \\
\hline Single parent families - divorced parents & $42 \cdot 3$ & $1 \cdot 05$ & $0.58,1.89$ & & \\
\hline Family size (number of family members) & - & 0.94 & $0 \cdot 77,1 \cdot 11$ & - & - \\
\hline
\end{tabular}

Ref., referent category; SES, socio-economic status.

${ }^{*}$ All OR were adjusted for annual family income, parent's nationality, residence ownership, child's primary caregiver, popularity score, gender and for the clustering effect of schools.

Cells in bold indicate statistically significant OR.

Regarding Greece, the prevalence of overweight and obesity reported in the present study was comparable to the prevalence reported by other recent studies conducted in children of similar age living in urban and semi-urban areas from southern $(29 \cdot 8 \%$ overweight and $14.9 \%$ obesity $)^{(1)}$, northern $(25 \cdot 3 \%$ overweight and $5.6 \%$ obesity) ${ }^{(15)}$, western $(34.3 \%$ overweight and $9 \cdot 6 \%$ obesity) ${ }^{(16)}$ and eastern (30.3\% overweight and 6.7\% obesity) ${ }^{(1)}$ parts of Greece. Similar to the present study, the prevalence of obesity was also found to be significantly higher in male than female subjects in almost all of the aforementioned studies. The IOTF cut-off points were used for screening children's overweight and obesity in all of these studies.

Certain socio-economic and demographic factors that have been reported by previous studies as correlates of childhood overweight ${ }^{(17,18)}$ were also tested in the present study. A lower-medium annual family income of $€ 12000-20000$ was associated with an increased likelihood of childhood overweight and obesity compared to a higher annual family income level of $€>30000$, both at the bivariate and multivariate levels. This finding is in line with those reported from other recent studies conducted in children in developed countries indicating that income, 
which is probably the most accurate index of socioeconomic status, is inversely related to the consumption of energy-dense foods, such as fats and sweets, full-fat meat, dairy products, etc. ${ }^{(19-21)}$. In contrast, children from relatively lower-income families tend to have a lower intake of vegetables, fruits and whole-grain products ${ }^{(19)}$. It has been reported that the energy density of foods and their energy cost are inversely related, which means that the more energy-dense diets represent the lowest-cost dietary options to the consumer ${ }^{(22,23)}$. Provided that higher socioeconomic status is probably reflected by residence ownership, then the finding of the present study regarding the association of this other index of socio-economic status with childhood overweight contradicts that observed for income. The increased likelihood of overweight observed in children whose parents owned their house could probably be ascribed to the fact that many parents reported their residence as privately owned, although their house was bought with a housing bank loan. In this case, residence ownership should not be considered as an accurate index of higher socio-economic status since a substantial amount of the family's budget pays off the monthly instalments of the bank loan, probably having a direct impact on children's nutrition. Moreover, residence ownership may not be a representative index of socio-economic status, when used as a single index ${ }^{(24)}$.

The significant positive association observed in the present study between residence ownership and childhood overweight and obesity could also be attributed to the mediating role of the parent's nationality. More specifically, our findings showed that Greek nationals were $9 \cdot 1$ times $(\mathrm{OR}=9 \cdot 09 ; 95 \% \mathrm{CI} 6 \cdot 18,13 \cdot 39)$ more likely to own their house and they were also $38 \%$ more likely to be overweight or obese than non-Greek nationals. The vast majority of non-Greek subjects, whose parents came to Greece as economic immigrants during the period of the economic and political transition in the 1990s, were from Albania (75.6\%) and from other Eastern European countries (i.e. Russia, Ukraine, Poland, Serbia, etc.; 12·6\%). In these countries, Lobstein and Frelut ${ }^{(3)}$ reported lower prevalence rates of childhood overweight in comparison to children living in the non-Eastern bloc European countries. The implication of this observation is that the lower prevalence of overweight also reported in the present study for non-Greek subjects, having mainly an Eastern European nationality, could be attributed to a genetic predisposition for lower BMI compared to Greek children. To our knowledge, no evidence exists on this topic, and therefore further research, including the study of certain genes and polymorphisms, is needed in order to shed more light on these differences.

Children's 'popularity' score was another index that was found to be inversely associated with childhood overweight when analysed at the bivariate level. A potential explanation for this finding might be the fact that children who are more athletic and of normal weight are usually more popular among their peers. Previous studies have also confirmed the association observed in the present study between weight status and social acceptance in populations of preadolescents. Reynolds et al. $^{(25)}$ have reported that overweight was an index of less social acceptance in girls aged 9-14 years. Furthermore, Latner and Stunkard ${ }^{(26)}$ have reported that overweight and obese (10-11 years old) children were significantly less popular than their normalweight peers. According to previous studies, children before and during puberty are very much concerned about their weight and body image ${ }^{(27)}$; thus, another way of explaining this association could be that children's 'popularity' concerns related to their body image may control the increase in their body weight.

Another important outcome of the regression analysis performed in the present study was that children whose primary caregiver was their grandmother had an increased likelihood of being overweight or obese both at the bivariate and multivariate levels. In a recent study, it was observed that food consumption in children was adjusted according to the stimuli induced by the food served by their caregivers or parents, instead of being regulated by the satiety and increased energy following the previously consumed meals ${ }^{(28)}$. In most cases, grandparents tend to believe that overweight signifies healthiness ${ }^{(29)}$ or that young children may grow out of being overweight ${ }^{(30)}$. Another interpretation of this particular finding is probably based on the so-called 'post-World War II overfeeding syndrome,(31) referring to the post-war period in Greece when there was severely decreased accessibility to food. Children's grandmothers, who most likely experienced hunger and extreme difficulties in finding food as children during those years, tend to overfeed their grandchildren with affluent portions of foods, as an expression of their care and insecurity. Furthermore, Gordon-Larsen et al. ${ }^{(7)}$, in a study of girls aged 6-9 years, observed that caregivers, including grandmothers, influenced their children in adopting a sedentary lifestyle, as they considered sedentary habits, such as television viewing, a more secure and easier way of having control of their children.

The present findings should be interpreted in the light of the limitations and strengths of the present study. Regarding the limitations, the cross-sectional design of the study does not allow the establishment of cause-effect relationships, but only generates hypotheses about the possible role of the social, economic and demographic factors examined in the present study on the development of overweight and obesity in children. Moreover, it should be noted that the present findings are the preliminary results of an ongoing study and thus should be interpreted with caution. However, the relatively large study sample and the random and multi-stage sampling of schools yielded in a representative sample of the wider region of Athens and this could be considered as a strength of the present study. Furthermore, the assessment of weight status in almost all children in the schools under study, combined with the 
fact that we observed no significant differences in the prevalence of overweight and obesity between 'study subjects' and 'weight and height participants' can also be considered as the strengths of the present study, indicating that our findings are unbiased and controlled for the parameter of will or motivation for participation in the study and its consequent possible confounding effect.

In conclusion, the present study showed that the considerably high prevalence of overweight and obesity among urban primary-school children in Greece also seems to have a socio-economic and demographic dimension. More specifically, lower family income and grandmother as the child's primary caregiver were the main risk factors for being overweight and obese among primary-school children living in a large urban area in Greece. The conclusion of the present study has several implications for the prevention of childhood obesity. Appropriate interventions and population-based strategies are needed, targeting lower-income families and probably those families in which both parents are working and the grandmother acts as the child's primary caregiver.

\section{Acknowledgements}

Source of funding: The Healthy Growth Study was supported with a research grant from the Friesland Foods Hellas. The present study has been facilitated by the EUfunded HOPE project 'Health promotion through Obesity Prevention across Europe (the Commission of the European Communities, SP5A-CT-2006-044128). The study does not necessarily reflect the Commission's views and in no way anticipates the Commission's future policy in this area. Conflicts of interest: The study sponsor had no role in the design of the study; the collection, analysis or interpretation of the data; the writing of the manuscript; and in the submission of the paper. None of the other authors had any personal/financial/potential conflict of interest. Y.M. worked as a part-time science and nutrition consultant for FrieslandCampina Hellas. Authorship responsibilities: Y.M. and G.M were responsible for the study design and supervision of the field study. Y.M., G.M. and O.A. performed the statistical analyses and the interpretation of the data. All authors carried out the data collection and contributed to data management, to database preparation and to the writing and approval of the final version of the manuscript submitted for publication.

\section{References}

1. Manios Y, Magkos F, Christakis G et al. (2005) Twenty-year dynamics in adiposity and blood lipids of Greek children: regional differences in Crete persist. Acta Paediatr 94, 859-865.

2. Cattaneo A, Monasta L, Stamatakis E et al. (2010) Overweight and obesity in infants and pre-school children in the European Union: a review of existing data. Obes Rev 11 , 389-398.
3. Lobstein $\mathrm{T} \&$ Frelut ML (2003) Prevalence of overweight among children in Europe. Obes Rev 4, 195-200.

4. Sobal J \& Stunkard AJ (1989) Socioeconomic status and obesity: a review of the literature. Psychol Bull 105, 260-275.

5. Lamerz A, Kuepper-Nybelen J, Wehle C et al. (2005) Social class, parental education, and obesity prevalence in a study of six-year-old children in Germany. Int J Obes (Lond) 29, 373-380.

6. Patrick H \& Nicklas TA (2005) A review of family and social determinants of children's eating patterns and diet quality. J Am Coll Nutr 24, 83-92.

7. Gordon-Larsen P, Adair LS \& Popkin BM (2003) The relationship of ethnicity, socioeconomic factors, and overweight in US adolescents. Obes Res 11, 121-129.

8. Serra-Majem L, Aranceta Bartrina J, Perez-Rodrigo C et al. (2006) Prevalence and determinants of obesity in Spanish children and young people. Br J Nutr 96, Suppl. 1, S67-S72.

9. Manios Y, Costarelli V, Kolotourou M et al. (2007) Prevalence of obesity in preschool Greek children, in relation to parental characteristics and region of residence. BMC Public Health 7, 178.

10. Apfelbacher CJ, Loerbroks A, Cairns J et al. (2008) Predictors of overweight and obesity in five to sevenyear-old children in Germany: results from cross-sectional studies. BMC Public Health 8, 171.

11. Shrewsbury V \& Wardle J (2008) Socioeconomic status and adiposity in childhood: a systematic review of crosssectional studies 1990-2005. Obesity 16, 275-284.

12. Sharma M (2006) School-based interventions for childhood and adolescent obesity. Obes Rev 7, 261-269.

13. University of Crete (1991) Food Composition Tables. http://nutrition.med.uoc.gr/greektables/

14. Lobstein TJ, James WP \& Cole TJ (2003) Increasing levels of excess weight among children in England. Int J Obes Relat Metab Disord 27, 1136-1138.

15. Krassas GE, Tzotzas T, Tsametis C et al. (2001) Prevalence and trends in overweight and obesity among children and adolescents in Thessaloniki, Greece. J Pediatr Endocrinol Metab 14, Suppl. 5, 1319-1326; discussion 1365.

16. Angelopoulos PD, Milionis HJ, Moschonis G et al. (2006) Relations between obesity and hypertension: preliminary data from a cross-sectional study in primary schoolchildren: the children study. Eur J Clin Nutr 60, 1226-1234.

17. Ferreira I, van der Horst K, Wendel-Vos W et al. (2007) Environmental correlates of physical activity in youth - a review and update. Obes Rev 8, 129-154.

18. Kimm SY \& Obarzanek E (2002) Childhood obesity: a new pandemic of the new millennium. Pediatrics 110, 1003-1007.

19. James WP, Nelson M, Ralph A et al. (1997) Socioeconomic determinants of health. The contribution of nutrition to inequalities in health. BMJ 314, 1545-1549.

20. Ruxton CH, Kirk TR, Belton NR et al. (1996) Relationships between social class, nutrient intake and dietary patterns in Edinburgh schoolchildren. Int J Food Sci Nutr 47, 341-349.

21. Mazur RE, Marquis GS \& Jensen HH (2003) Diet and food insufficiency among Hispanic youths: acculturation and socioeconomic factors in the third National Health and Nutrition Examination survey. Am J Clin Nutr 78, 1120-1127.

22. Drewnowski A \& Darmon N (2005) The economics of obesity: dietary energy density and energy cost. Am J Clin Nutr 82, 265S-273S.

23. Drewnowski A \& Specter SE (2004) Poverty and obesity: the role of energy density and energy costs. Am J Clin Nutr 79, 6-16. 
24. Krieger N, Williams DR \& Moss NE (1997) Measuring social class in US public health research: concepts, methodologies, and guidelines. Annu Rev Public Health 18, 341-378.

25. Reynolds KD, Franklin FA, Leviton LC et al. (2000) Methods, results, and lessons learned from process evaluation of the high 5 school-based nutrition intervention. Health Educ Behav 27, 177-186.

26. Latner JD \& Stunkard AJ (2003) Getting worse: the stigmatization of obese children. Obes Res 11, 452-456.

27. Schur EA, Sanders M \& Steiner H (2000) Body dissatisfaction and dieting in young children. Int J Eat Disord 27, 74-82.
28. Mrdjenovic G \& Levitsky DA (2005) Children eat what they are served: the imprecise regulation of energy intake. Appetite 44, 273-282.

29. Adams AK, Quinn RA \& Prince RJ (2005) Low recognition of childhood overweight and disease risk among NativeAmerican caregivers. Obes Res 13, 146-152.

30. Etelson D, Brand DA, Patrick PA et al. (2003) Childhood obesity: do parents recognize this health risk? Obes Res 11, 1362-1368.

31. Mamalakis G \& Kafatos A (1996) Prevalence of obesity in Greece. Int J Obes Relat Metab Disord 20, 488-492. 